\title{
Fluorescence as a Tool to Study Lipid-Protein Interactions: The Case of $\alpha$-Synuclein
}

\author{
Azucena Gonzalez-Horta*, Brenda Gonzalez Hernandez, Abelardo Chavez-Montes \\ Faculty of Biology, University Autonoma de Nuevo Leon, San Nicolás de los Garza, Mexico \\ Email: " azucena.gonzalezhr@uanl.edu.mx
}

Received December 25, 2012; revised January 27, 2013; accepted February 8, 2013

\begin{abstract}
During the past 20 years there has been a remarkable growth in the use of fluorescence in the biological sciences. Fluorescence is now a dominant methodology used extensively in biochemistry, biophysics, biotechnology, medical diagnostics, flow cytometry, DNA sequencing and genetic analysis to name a few. It is one of the most powerful methods to study protein folding, dynamics, assembly and interactions as well as membrane structure. $\alpha$-Synuclein belongs to the class of intrinsically disordered proteins lacking of a well-folded structure under physiological conditions. The conversion of $\alpha$-synuclein from a soluble monomer to an insoluble fibril may underlie the neurodegeneration associated with Parkinson's disease (PD). Although the exact mechanism of $\alpha$-synuclein toxicity is still unknown, it has been proposed that disturbs membrane structure, leading to increased membrane permeability and eventual cell death. This review highlights the significant role played by fluorescence techniques in unraveling the nature of interactions between $\alpha$-synuclein and membranes and its implications in PD.
\end{abstract}

Keywords: Fluorescence Spectroscopy; Lipid-Protein Interactions; $\alpha$-Synuclein

\section{Introduction}

Fluorescence spectroscopy-based techniques using conventional fluorimeters have been extensively applied since the late 1960s to study different aspects of membrane-related phenomena, i.e., mainly relating to lipidlipid and lipid-protein (peptide) interactions. These types of studies encompass measurements of fluorescence excitation and emission spectra, fluorescence time decays (lifetimes) and fluorescence polarization (or anisotropy) using of a large variety of fluorescent probes [1,2]. The ability of $\alpha$-synuclein to exchange between many different conformational states-disordered monomer, partially structured oligomer, $\alpha$-helical membrane-bound monomer, and $\alpha$-sheet fibrillar aggregate-may be central to its native function as well as to its role in Parkinson's disease (PD). $\alpha$-Synuclein is the primary component of Lewy bodies, dense cytoplasmic amyloid inclusions, which are associated with selective loss of dopaminergic neurons in the substantia nigra region of the brain. Although its precise role in the progression of PD remains unclear, point mutations (A30P, E46K and A53T) and multipliction of the $\alpha$-synuclein gene linked to familial forms of PD established that it is crucial to disease development. Commonly used fluorescent techniques, include intrinsic and extrinsic fluorophores,

${ }^{*}$ Corresponding author. fluorescent probes incorporated in the membrane, steady-state and lifetime measurements of fluorescence emision, fluorescence resonance energy transfer (FRET) and fluorescence microscopy, have been employed to monitor the kinetics of aggregation of $\alpha$-synuclein, its distinct phospholipid vesicle and micelle interactions as well as its membrane-perturbation properties to contribute to our understanding of its native function and its role in Parkinson's disease.

\section{Fluorescence Spectroscopy}

\subsection{Definition and General Characteristics}

Fluorescence is a molecular phenomenon in which a substance absorbs light, then radiate part of this absorbed energy as light of another color, one of lower energy and thus longer wavelength. This process is known as excitation and emission. The shift from excitation wavelength to emission wavelength is called the Stokes shift. The ability of a fluorophore to absorb encountered light is known as the extinction coefficient. Once energy is absorbed, the fluorophore has some probability less than one of releasing this absorbed energy as light. This characteristic is called the Quantum yield (number of emitted photons relative to the number of absorbed photons) $[3,4]$. Substances with the largest quantum yields, approaching unity, display the brightest emissions. The lifetime is also 
important, as it determines the time available for the fluorophore to interact with or diffuse in its environment, and hence the information available from its emission. Together, these properties dictate the basic fluorescent properties (brightness and spectra) of an individual fluorescent dye. The power of fluorescence spectroscopy lies on its broad applicability. Almost all proteins have natural fluorophores, tyrosine and tryptophan residues, which allow study of changes in protein conformation. Also site-specific labeling with external fluorophores is easily achievable by mutagenesis and chemical modifications. Another advantage is that fluorescence spectroscopy requieres a small amount of material (pM-nM range) and has a high signal-to noise ratio [3]. All these features make fluorescence spectroscopy an invaluable technique for studying protein function.

\subsection{Intrinsic Fluorophores}

Tryptophan (Trp) and tyrosine (Tyr) residues are naturally occurring fluorophores in proteins (Figure 1). Trp has the highest quantum yield and its emission maximum is sensitive to the polarity of its environment; conesquently, Trp is a commonly used intrinsic fluorescent probe in studying protein folding and dynamics. The $\lambda_{\max }$ of a buried Trp is around $335 \mathrm{~nm}$, whereas fully solvent accesible Trp has $\lambda_{\max }$ around $355 \mathrm{~nm}$. Trp fluorescence lifetime measurements can overcome the limitations of steady-state measurements by allowing determination of the different lifetimes or anisotropy of different residues $[3,5]$. Tyr has significantly lower quantum yield than Trp and is usually only used as an intrinsic fluorescent probe in Trp-lacking proteins or peptides, since energy transfer to Trp residues usually quenches the Tyr fluorescence. The Tyr emission maximum is near $305 \mathrm{~nm}$ and is not sensitive to the polarity of environment; however, Tyr emission depends on $\mathrm{pH}$. The phenolic hydroxyl group has a $\mathrm{pKa}$ near 10 and at high $\mathrm{pH}$ is deprotonated. The deprotonation results in the formation of tyrosinate, which has a red shifted emission spectrum $\left(\lambda_{\max } 340 \mathrm{~nm}\right)$. Phe has a very low quantum yield, and is usually not used as a fluorescent probe. The close proximity of Phe<smiles>N[C@@H](Cc1c[nH]c2ccccc12)C(=O)O</smiles><smiles>NC(Cc1ccc(O)cc1)C(=O)O</smiles><smiles>NC(Cc1ccccc1)C(=O)O</smiles>

Figure 1. Intrinsic biochemical fluorophores in proteins. to Tyr or Trp, and Tyr to Trp, can influence the quantum yield of Trp and Tyr through energy transfer [3].

\subsection{Extrinsic Fluorophores}

In many cases, the intrinsic fluorescence of the protein/ peptide does not exist or is not fluorescent in a convenient region of the UV visible spectrum. It is very common to create single Trp mutants or to attach covalently a fluorescent group to a cysteine residue. With this approach, it is posible to explore the environment around different regions of a protein, by different fluorescent methodologies, obtaining a large amount of information [6]. A wide variety of extrinsic probes have been developed for labeling the macromolecules in such cases. Two of the most widely used probes, dansyl chloride (DNS-Cl) which stands for 1-dimetylamino-5-naphthylsulfonyl chloride, and fluorescein isothiocyanate (FITC) are shown in Figure 2. These probes react with the free amino groups of proteins, resulting in proteins that fluoresce at blue (DNS) [7] or green (FITC) wavelengths. Proteins can also be labeled on free sulfhydryl groups using maleimide reagents such as BODIPYmaleimide. It is frequently useful to use longer-wavelength probes such fluorophores from the Rodhamine family (i.e., Texas Red) [3].

\subsection{Membrane Fluorescent Dyes}

Lipids are amphiphilic in nature and consist of a hydrophobic chain and a hydrophilic head group region. Accordingly, there are in principle two regions within the molecule to which fluorescent dyes can be covalently coupled. The intramolecular localization of the dye as well as the chemical nature of the dye itself are highly relevant, as they will determine the biophysical properties of the final fluorescent lipid analog as such, which in turn are relevant for the assay in which the probes will be eventually used. The majority of the fluorescent dyes that have been used in lipid derivatization, especially those emitting in the visible light region, are hydrophilic. When they are attached in the chain region, the dye will change the hydrophilic/hydrophobic balance of the lipid molecule [8]. Alternatively, head group labeled lipids can also be prepared when properties of the chain regions are important, e.g., for phase determination in biological membranes. DPH, 1,6-diphenyl-1,3,4-hexatriene, is one of the most commonly used membrane probes. Addition of DPH to a membrane suspension results in complete binding, with no significant emission from DPH in the aqueous phase. All the emission from DPH is then due to DPH in the membrane environment. There are a wide variety of lipid probes which can be attached to the fatty acid chains or to the phospholipids themselves. Figure 3 shows a few examples of these dyes [3]. Membranes can 
<smiles>CN(C)c1cccc2c(S(=O)(=O)Cl)cccc12</smiles>

DNS-Cl

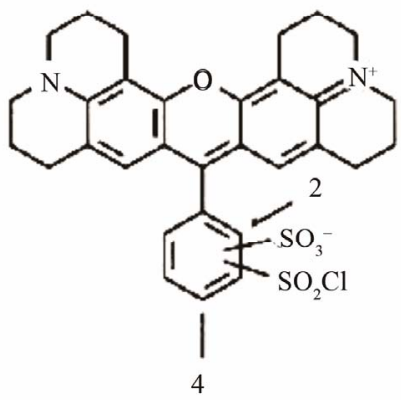

Texas Red sulfonyl chloride<smiles>O=C(O)c1cc(N=C=S)ccc1-c1c2ccc(=O)cc-2oc2cc(O)ccc12</smiles><smiles></smiles>

BODIPY 499/508 malcimide

Figure 2. Fluorophores for covalent labeling of biomolecules.

Headgroup labeled

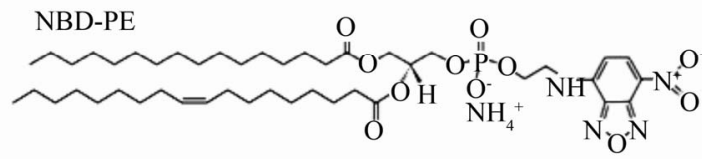

Pyrene-PF
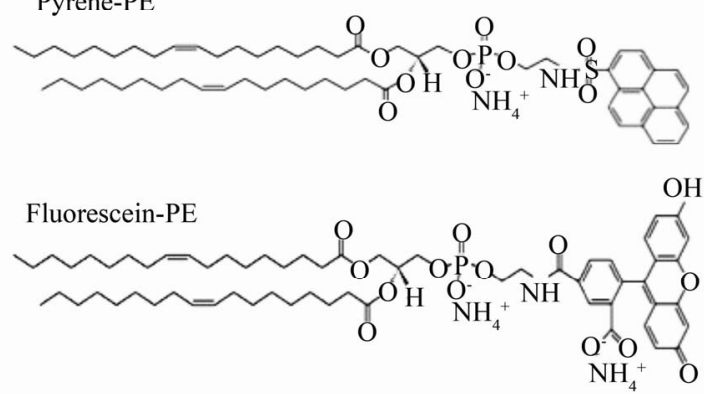

Fatty acid labeled
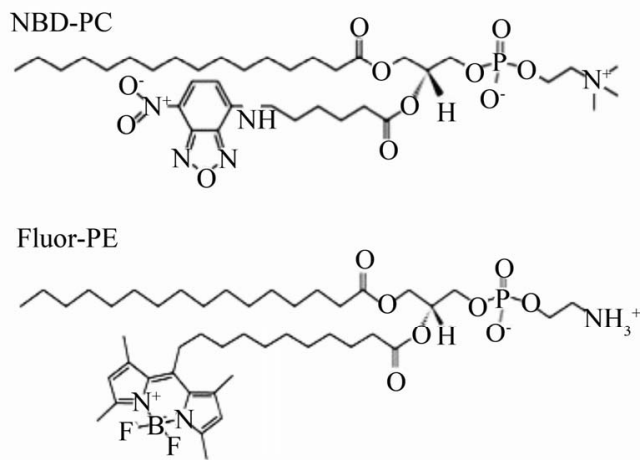

Figure 3. Fluorescent phospholipid analogues. $\mathrm{PC}=$ phosphatidylcholine; $\mathrm{PE}=$ phosphatidylethanolamine. 
also be labeled by covalent attachment of probes to the lipids. This is useful with more water-soluble probes like fluorescein or rhodamine. The probes can be forced to localize in the membrane by attachment to long acyl chains or to the phospholipids themselves. Depending on chemical structure, the fluorescent group can be positioned either on the fatty acid side chains (Fluorenyl-PC) or at the membrane-water interface (TexasRed-PE) (Figure 3). Membrane fluorescent probes are used for stuying lateral diffusion, fluidity, positioning of lipids and proteins in the lipid bilayer and surface potential of membranes. DPH serves as a sensor of the disordering of fatty acyl chains that occurs during the transition from gel to liquid crystalline phase. This dye also detects the perturbation of the fatty acyl chain packing upon insertion of proteins [5]. The dansyl group and 2-N-4-nitrobenzo-2-oxa-1,3-diazole (NBD) attached to the phospholipid head group are responsive to surface-solvent effects. NBD is sensitive to the environmental polarity, it is weakly fluorescent in water and strongly fluorescent in hydrophobic environments. Dansylated lipids have been used to study solvent relaxation process in membranes.

\section{3. $\alpha$-Synuclein Interaction with Lipid Membranes}

\subsection{Alpha-Synuclein and Parkinson's Disease}

Parkinson's disease (PD) is the second most common neurodegenerative disease, affecting $1 \%-2 \%$ of the population over age 65 . It involves loss of dopaminergic neurons in the substantia nigra, which leads to decreased dopamine levels in the striatum, causing symptom such as tremors, rigidity in muscles and bradykinesia [11-13]. While the exact cause of PD is not known, aggregation of the presynaptic protein $\alpha$-synuclein is believed to be a critical role in the etiology of the disease. There is a growing number of cellular toxicity studies showing that oligomers have a higher cytotoxicity compared to the fibrillar form of the proteins [14-17], suggesting that soluble amyloid oligomers may be the cause of cellular toxicity instead of the fibrillar aggregates [15,18-21]. One conceivable pathway to cellular toxicity would be the permeabilization of cellular membranes by oligomers. However, the mechanisms by which oligomers cause bilayer permeabilization still remain unclear. On the one hand it has been hypothesized that $\alpha$-synuclein oligomers are able to form pore-like structures mediating the permeation of small molecules [2023 ] whereas on the other hand membrane thinning by the interaction with the oligomeric protein, which increases the permeability of the phospholipid bilayers has been postulated. In the case of $\alpha$-synuclein, the first reports on membrane permeabilization by pore-like, annular shaped oligomers are from the Lansbury group in the early 2000s [22,23]. While the AFM images obtained by this group suggest pore-like mechanism, quantitative biophysical information on the structures and composition of these oligomeric aggregation intermediates is lacking, for this reason several research groups have been employed various techniques including fluorescence spectroscopy to understand the mechanism by which $\alpha$-synuclein is implicated in PD. Native alpha synuclein is a small protein, $14 \mathrm{kDa}$, which analysis of the primary structure shows three different regions: the N-terminal region consisting of five degenerate 11-mer repeats KTKEGV of overall positive charge, the hydrophobic central part (known as the NAC region) and the C terminal 40 residues which are highly enriched in negatively charged amino acids (Figure 4). There are two phenylalanine and four tyrosine residues in the protein, localized in distinct regions; Phe4 and Tyr39 are located in the N-terminal region, Phe94 at the end of the central segment and Tyr125, Tyr133 and Tyr136 at the end of the C-terminal region. There are no tryptophan residues [23,24]. In aqueous solution $\alpha$-synuclein adopts a random coil conformation [25], but in the presence of negatively charged phospholipid vesicles, the N-terminal residues-1-100 have been shown to facilitate lipid binding by adopting an $\alpha$-helical conformation [26,27] (Figure 4). In vitro, $\alpha$-synuclein can aggregate into amyloid-like fibrils rich in $\alpha$-sheet structure that resemble those found in neuronal protein deposits in vivo [28].

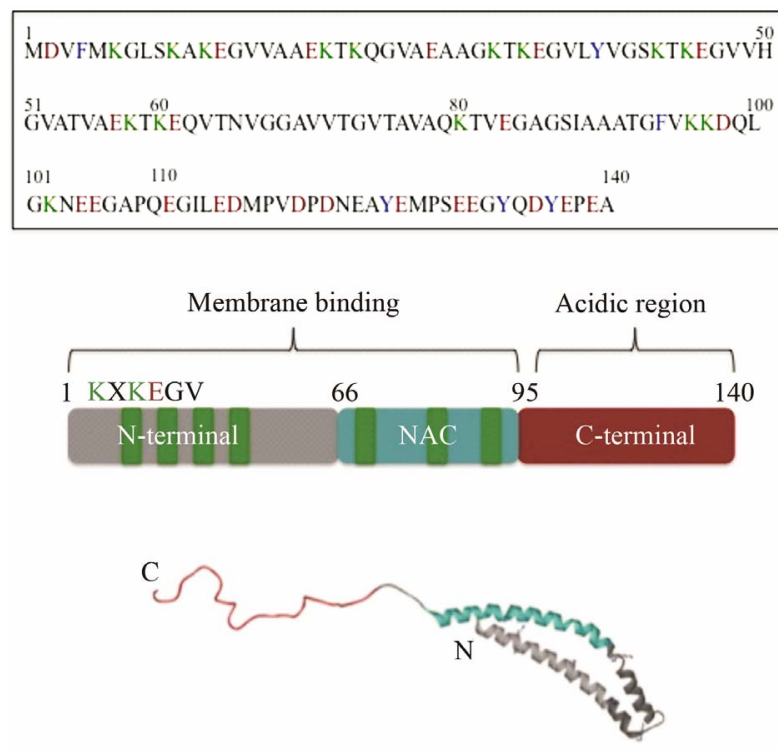

Figure 4. (Top) $\alpha$-Synuclein primary amino acid sequence with aromatic (blue), acidic (red) and lysine (green) residues highlighted. (Middle) Schematic representation of three different regions of $\alpha$-synuclein. (Bottom) Side view of micelle-bound $\alpha$-synuclein (PDB ID: 1XQ8). The membrane binding domain is colored in gray. The NAC region is showing in blue and the acidc region in red. 


\subsection{Intrinsic Tyrosine Fluorescence}

Monitoring intrinsic Tyr fluorescence of $\alpha$-synuclein during fibril formation or interaction with lipid vesicles revealed changes in the shape and intensity of the Tyr emission spectrum which is manifested by a shift in the emission maximum from 305 to $340 \mathrm{~nm}$. Th estructural transition occurs in the $\mathrm{N}$-terminal and central parts of the protein, whereas the $\mathrm{C}$-terminal remains unfolded.

Fink et al., (2003) characterized the binding of $\alpha$ synuclein to vesicles of different compositions by measuring the change in intrinsic fluorescence emanating from the four Tyr residues in the protein as a function of lipid concentration. They observed that $\alpha$-synuclein can bind to the surface of PC vesicles, but not as strong as with $\mathrm{PA} / \mathrm{PC}$ or $\mathrm{PG} / \mathrm{PC}$. This suggest that electrostatic interactions play an important role in the binding of protein to lipid, however the binding was also observed at very high ionic strengths, showing that additional forces, presumably hydrophobic, must also contribute significantly to the interaction of protein and the lipid. It is the hydrophobic interactions, because of the helical conformation, that lead to the critical penetration of the bilayer. Using NBD- and Laurdan-labeled vesicles they also demonstrated that soluble monomeric $\alpha$-synuclein and fibrillar $\alpha$-synuclein can insert into the bilayer to a depth of several angstroms, and affect membrane properties. With a dye release assay they could observe the ability of, its protofibrils and fibrils to disrupt lipid membranes. The results clearly show that fibrillar $\alpha$-synuclein has a much higer ability to disrupt membrane bilayers than soluble monomeric $\alpha$-synuclein, and the order of effectiveness in disruption is $\mathrm{PA}>\mathrm{PG}>\mathrm{PC}$, which is consistent with the membrane affinity [29]. These observations suggest a potential source of neurotoxicity for this protein, namely via disruption of membranes leading to ion fluxes that would be lethal to the cell.

\subsection{Fluorescence of Tryptophan Mutants of $\alpha$-Synuclein}

Because the $\lambda_{\max }$ of tyrosine is insensitive to its environment several tryptophan mutants have been constructed as fluorescence probes. Incorporation of Trp into $\alpha$-synuclein permits the use of a variety of probes, includig solvent accessibility probes such as intrinsic fluorescence emission and acrylamide quenching, to analyze the presence of oligomers and the kinetics of their formation via fluorescence anisotropy and changes in compactness via FRET $[9,10]$. Investigations of Y39W and Y125W mutants show that both tryptophans are exposed to solvent in monomeric unfolded $\alpha$-synuclein. During fibril formation Trp39 becomes partially buried in the core of the fibrils as revealed by a blue shift in $\lambda_{\max }$ (340 $\mathrm{nm}$ ), whereas Trp125 remains solvent exposed. Intere- stingly, at acidic $\mathrm{pH}$ the emission maxima of $\mathrm{Y} 39 \mathrm{~W}$ and Y125W are 345 and $345.5 \mathrm{~nm}$, indicating the hydrophobic collapse of $\alpha$-synuclein under acidic conditions, presumably due to loss of the negative charges in the Cterminal region, and consistent with previous studies showing a partially folded intermediate at acidic $\mathrm{pH}$ [30]. In a more recent work Pfefferkorn and Lee (2010) investtigated membrane-induced conformational changes by fluorescence as well as circular dichroism (CD) spectroscopy. Trp was substituted at four different aromatic residues (F4W, Y39W, F94W and Y125W) determined the crucial protein-to membrane conditions and key sites of interaction that promote protein aggregation. They demonstrated that Trp fluorescence is a sensitive sitespecific probe of $\alpha$-synuclein interactions with both liposomes and micelles. They estimate that the protein binds to POPA:POPC vesicles with a relatively low surface coverage of $-7 \%$ ( -120 proteins for a $80 \mathrm{~nm}$ diameter vesicle) at saturation. Trp at position 4 and 94 were the most sensitive to the lipid bilayer with pronounced spectral blue-shifts (Trp4: $\Delta \lambda_{\max }-23 \mathrm{~nm}$; Trp $94: \Delta \lambda_{\max }-10$ $\mathrm{nm})$ and quantum yield increases (Trp4, Trp94-3 fold) while Trp39 and Trp125 remain primarily water-exposed. All sites interact with the membrane except Trp125 [31].

\subsection{Förester Resonance Energy Transfer (FRET) Studies}

FRET measurements were used to study the structure and dynamics of $\alpha$-synuclein in three different conditions: at

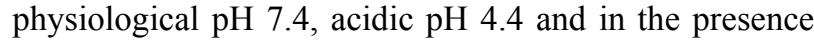
of SDS micelles [32]. Tryptophan and 3-nitrotyrosine at six different locations in the protein were chosen as donor-acceptor pairs with a Förster critical distance of $26 \AA$. The results show that $\alpha$-synuclein exists as ensamble of compact and extended conformations in all tested conditions. In acidic solutions, the $\mathrm{C}$-terminal of $\alpha$-synuclein becomes more compact, whereas in the presence of SDS micelles the $\mathrm{N}$-terminal is more compact and the $\mathrm{C}$-terminal is more extended. Fluorescence resonance energy transfer was also used to analyze the conformational changes of $\alpha$-synuclein during aggregation [9]. The triple mutant Y125W, Y133F, Y136F of $\alpha$-syuclein has one tyrosine-tryptophan donor-acceptor pair with a Förester critical distance of $15 \AA$. The calculated distance between Y39 and W125 in monomeric $\alpha$-synuclein is $25 \AA$, which decreases during aggregation to $22 \AA$ (early oligomeric species) and $19 \AA$ (late oligomeric species).

\subsection{Vesicle Permeabilization by Alpha-Synuclein}

Membrane permeabilization by $\alpha$-synuclein oligomers may play a major role in the pathological mechanism of $\mathrm{PD}$, potentially due to the impairment of membranous cellular structures such as mitochondria and synaptic vesicles $[33,34]$. The first decisive step for membrane 
permeabilization is the recruitment of oligomers to the lipid bilayer. This process dependent on the presence of negatively charged lipids being in a liquid disordered phase, while the headgroup is only of minor importance $[35,36]$. This behavior, which is comparable to monomeric $\alpha$-synuclein suggests that mainly electrostatic interactions between the positively charged core of the oligomers and negatively charged lipid headgroups cause membrane binding [37,38]. In order to elucidate the mechanisms by which the $\alpha$-synuclein oligomers permeabilize lipid bilayers, Subramaniam group tested different lipid mixtures in leakage experiments either based on the release of calcein from large unilamellar vesicles (LUVs) containing self-quenching concentrations of the probe or on measuring the influx of dithionite into LUVs labeled with 1-palmitoyl-2-6-[(7-nitro-2-1,3-benzoxadiazol-4-yl) amino] hexanoyl-phosphatidylcholine $\left(\mathrm{C}_{6}\right.$-NBD-PC). While for LUVs prepared from palmitoyl-oleoyl-phosphatidylcholine (POPC) no leakage occurred, a strong disruption of the vesicles could be observed for LUVs consisting of the negatively charged lipids di-oleoylphosphatidylserine (DOPS), palmitoyl-oleoyl-phosphatidylglycerol (POPG) or soy phosphatidylinositol (PI). They also observed a slight influence of the lipid headgroup [39]. Kinetic measurements showed that the effected LUVs became permeable within minutes. However for LUVs containing POPC (1:1 molar ratio) no membrane permeabilization by $\alpha$-synuclein oligomers was observed in calcein release assays. More detailed experiments based on the bleaching of C6-NBD-PC revealed the existence of a sharp cutoff between 70 and $90 \mathrm{~mol} \%$ of POPG for POPC/ POPG mixtures where membrane permeabilization sets in [40], suggesting that while the mere presence of negatively charged lipids is sufficient to trigger membrane binding of $\alpha$-synuclein oligomers, only membranes containing high fractions of these lipids are susceptible to permeabilization. They speculate that disturbed lipid packing due to the repulsion between equally charged lipid molcules is decisive. Comparing membrane permeabilization of LUVs prepared from di-oleoyl-phosphatidylglycerol (DOPG), POPG and mixture containing POPG/ colesterol (3:1 molar ratio) where the degree of lipid packing increases in ascending order, calcein release assays revealed that an increased membrane order is accompanied by a decreased membrane permeabilization [39]. The degree of lipid packing plays an important role in the permeabilization of membranes, since at least a partial exposure of the hydrophobic core seems to be a prerequisite for the permeabilization of phospholipid bilayers.

\subsection{Fluorescence Microscopy Methods}

To investigate and visualize directly the lipid- and domain-specific association of $\alpha$-synuclein with membranes, Herrmann et al. (2008) studied the binding of fluores- cently labeled $\alpha$-synuclein to giant unilamellar vesicles (GUVs) of different composition labeled with $1 \mathrm{~mol} \%$ of the green fluorescent lipid analogue 1-palmitoyl-2-[6-[(7nitro-2-1,3-benzox-adiazol-4-yl)amino]hexanoyl]-sn-glycero-3-phosphocholine $\left(\mathrm{C}_{6}\right.$-NBD-PC), which is known to localize preferentially to the liquid-disorder (Ld) phase [41]. They observed that phospholipids with anionic head groups are required for the interaction of the protein with membranes. While the degree of saturation of the acyl chains of these lipids plays only a minor role. Liquidordered (Lo) domains are not a target of $\alpha$-synuclein even in the presence of negatively charged lipids. Hence, both lipid packing and ionic interaction are important determinants of the association of $\alpha$-synuclein with membranes [42].

In order to gain more insight into the mechanism of lipid bilayer disruption by $\alpha$-synuclein oligomer species, Subramaniam group have used confocal fluorescence microscopy to observe the oligomer induced membrane permeability of giant unilamellar vesicles. They prepared Rhodamine labeled POPG GUVs encapsulating the dye 8-hydroxypyrene-1,3,6-trisulfonic acid (HPTS). To reduce the signal from un-encapsulated HPTS they added the quencher p-Xilene-bis, N-pyridinium bromide (DPX) to the solution outside the GUVs. When oligomeric $\alpha$-synuclein was added to the imaging chamber, the fluorescence from the vesicle interior was lost. The images show that the leakage proces is fast and that the vesicles appear morphologically unchanged. Results from DLS experiments confirm that LUVs stay largely intact upon oligomer interaction [43]. These results, combined with work form other groups suggest that $\alpha$-synuclein oligomers do not necessarily form pore-like sructures. The emerging consensus is that local structural rearrangements of the protein lead to insertion of specific regions into the hydrophobic core of the lipid bilayer, thereby disrupting the lipid packing.

\section{Conclusion}

Fluorescence spectroscopy can be applied to a wide range of problems in the chemical and biological sciences. The measurements can provide information on a wide range of molecular process, including the interactions of solvent molecules with fluorophores, rotational diffusion of biomolecules, distances between sites on biomolecules, conformational changes, and binding interactions. The usefulness of fluorescence is being expanded by advances in technolgy for celular imaging and single-molecule detection. These advances in fluorescence technology are decreasing the cost and complexity of previously complex instruments. Fluorescence spectroscopy will continue to contribute to rapid advances in biology, biotechnology and nanotechnology. With regard 
to alpha-synuclein, fluorescence techniques have allowed characterization of the interaction of this protein and its various aggregated states with membranes of different types and show that oligomeric and fibrillar forms of the protein cause substantial perturbation of membranes and likely promote membrane permeability leading to cytotoxicity. In addition, elucidating the underlying mechanisms by which the $\alpha$-synuclein oligomers are able to penetrate the phospholipid bilayer will give valuable insights into their mode of action and presumably facilitate the development of a possible intervention strategy.

\section{Acknowledgements}

A.G.H. would like to acknowledge the support of PAICYT (CN416-10) Universidad Autonoma de Nuevo Leon. The authors would also like to acknowledge the contribution of a large number of authors who have contributed to the elucidation of alpha-synuclein interaction with membranes.

\section{REFERENCES}

[1] L. A. Bagatolli, "Membranes and Fluorescence Microscopy," Reviews in Fluorescence, Vol. 4, No. 1, 2007, pp. 33-51.

[2] J. R. Silvius and I. R. Nabi, "Fluorescence-Quenching and Resonance Energy Transfer Studies of Lipid Microdomains in Model and Biological Membranes," Molecular Membrane Biology, Vol. 23, No. 1, 2006, pp. 5-16. doi:10.1080/09687860500473002

[3] J. R. Lakowicz, "Principles of Fluorescence Spectroscopy," 3rd Edition, New York, Springer, 2006. doi:10.1007/978-0-387-46312-4

[4] J. W. Taraska, 'Mapping Membrane Protein Structure with Fluorescence," Current Opinion in Structural Biology, Vol. 22, No. 4, 2012, pp. 507-513. doi:10.1016/i.sbi.2012.02.004

[5] L. A. Munishkina and A. L. Fink, "Fluorescence as a Method to Reveal Structures and Membrane-Interactions of Amyloidogenic Proteins," BBA, Vol. 1768, No. 8, 2007, pp. 1862-1885. doi:10.1016/j.bbamem.2007.03.015

[6] C. M. Reyes, R. F. de Almeida, L. M. S. Loura and M. Prieto, "From Lipid Phases to Membrane Protein Organization: Fluorescence Methodologies in the Study of LipidProtein Interactions," In: C. R. Mateo, et al., Ed., Springer Series in Biophysics, Protein-Lipid Interactions SpringerVerlag Berlin Heidelgerg, Berlin, Vol. 9, 2006.

[7] I. Plasencia, A. Cruz, C. Casals and J. Perez-Gil, "Superficial Disposition of the N-Terminal Region of the Surfactant Protein SP-C and the Absence of Specific SP-BSP-C Interactions in Phospholipid Bilayers," Biochemical Journal, Vol. 359, 2001, pp. 651-659. doi:10.1042/0264-6021:3590651

[8] O. Maier, V. Oberle and D. Hoekstra, "Fluorescent Lipid Probes: Some Properties and Applications (A Review)," Chemistry and Physics of Lipids, Vol. 116, No. 1-2, 2002, pp. 3-18. doi:10.1016/S0009-3084(02)00017-8

[9] J. Kaylor, N. Bodner, S. Edridge, G. Yamin, D. P. Hong and A. L. Fink, "Characterization of Oligomeric Intermediates in Alpha-Synuclein Fibrillation FRET Studies of Y125W/Y133F/Y136F Alpha-Synuclein," Journal of Molecular Biology, Vol. 353, No. 2, 2005, pp. 357-372. doi:10.1016/j.jmb.2005.08.046

[10] A. Dusa, J. Kaylor, S. Edridge, N. Bodner, D. P. Hong and A. L. Fink, "Characterization of Oligomers during Alpha-Synuclein Aggregation Using Intrinsic Tryptophan Fluorescence," Biochemistry, Vol. 45, No. 8, 2006, pp. 2752-2760. doi:10.1021/bi051426z

[11] K. L. Lim, F. L. Dawson and T. M. Dawson, "The Best of Molecular Characters in Parkinson's Disease: Felons, Conspirators and Suspects," Annals of the New York Academy of Sciences, Vol. 991, No. 1, 2003, pp. 80-92. doi:10.1111/j.1749-6632.2003.tb07465.x

[12] A. Siderowf and M. Stern, "Update on Parkinson Disease," Annals of Internal Medicine, Vol. 138, No. 8, 2003, pp. 651-658.

[13] T. T. Warner and A. H. Schapira, "Genetic and Environmental Factors in the Cause of Parkinson's Disease," Annals of Neurology, Vol. 53, Suppl. 3, 2003, pp. S16S23. doi:10.1002/ana.10487

[14] A. Demuro, E. Mina, R. Kayed, S. C. Milton, I. Parker and C. G. Glabe, "Calcium Dysregulation and Membrane Disrpution as Ubiquitous Neurotoxic Mechanism of Soluble Amyloid Oligomers," The Journal of Biological Chemistry, Vol. 280, No. 17, 2005, pp. 17294-17300. doi:10.1074/jbc.M500997200

[15] A. L. Fink, "The Aggregation and Fibrillation of AlphaSynuclein," Accounts of Chemical Research, Vol. 39, No. 9, 2006, pp. 628-634. doi:10.1021/ar050073t

[16] R. Kayed, E. Head, J. L. Thompson, S. C. Milton, C. W. Cotman and C. G. Glabe, "Common Structure of Soluble Amyloid Oligomers Implies Common Mechanism of Pathogenesis," Science, Vol. 300, No. 5618, 2003, pp. 486489. doi:10.1126/science. 1079469

[17] M. J. Volles and P. T. Lansbury Jr., "Zeroing in on the Pathogenic Form of Alpha-Synuclein and Its Mechanism of Neurotoxicity in Parkinson's Disease," Biochemistry, Vol. 42, No. 26, 2003, pp. 7871-7878. doi:10.1021/bi030086j

[18] K. M. Danzer, D. Haasen, A. R. Karow, S. Moussaud, M. Habeck, A. Giese, H. Kretzschmar, B. Hengerer and M. Kostka, "Different Species of Alpha-Synuclein Oligomers Induce Calcium Influx and Seeding," The Journal of Neuroscience, Vol. 27, No. 34, 2007, pp. 9220-9232. doi:10.1523/JNEUROSCI.2617-07.2007

[19] M. S. Goldberg and P. T. Lansbury, "Is There a CauseAnd-Effect Relationship between Alpha-Synuclein Fibrillization and Parkinson's Disease?" Nature Cell Biology, Vol. 2, No. 7, 2000, pp. E115-E119. doi: $10.1038 / 35041081$

[20] H. Y. Kim, et al., "Structural Properties of Pore-Forming Oligomers of Synuclein," Journal of the American Chemical Society, Vol. 131, No. 47, 2009, pp. 17482-17489. doi:10.1021/ja9077599

[21] H. A. Lashuel, D. Hartley, B. M. Petre, T. Walz and P. T. 
Lansbury, "Neurodegenerative Disease-Amyloid Pores from Pathogenic Mutations," Nature, Vol. 418, No. 6895, 2002, pp. 291-300. doi:10.1038/418291a

[22] M. J. Volles and P. T. Lansbury Jr., "Vesicle Permeabilization by Protofibrillar Alpha-Synuclein Is Sensitive to Parkinson's Disease-Linked Mutations and Occurs by a Pore-Like Mechanism," Biochemistry, Vol. 41, No. 14, 2002, pp. 4595-4602. doi:10.1021/bi0121353

[23] H. A. Lashuel, B. M. Petre, J. Wall, M. Simon, R. J. Nowak, T. Walz and P. T. Lansbury Jr., "Alpha-Synuclein, Especially the Parkinson's Disease-Associated Mutants Forms Pore-Like Annular and Tubular Protofibrils," Journal of Molecular Biology, Vol. 322, No. 5, 2002, pp. 10891102. doi:10.1016/S0022-2836(02)00735-0

[24] L. Maroteaux, J. T. Campanelli and R. H. Scheller, "Synuclein: A Neuron Specific Protein Localized to the Nucleus and Presynaptic Nerve Terminal," The Journal of Neuroscience, Vol. 8, No. 8, 1988, pp. 2804-2815.

[25] P. H. Weinreb, W. Zhen, A. W. Poon, K. A. Conway and P. T. Lansbury Jr., "NACP a Protein Implicated in Alzheimer's Disease and Learning Is Natively Unfolded," Biochemistry, Vol. 35, No. 43, 1996, pp. 13709-13715. doi:10.1021/bi961799n

[26] R. Bussell, T. F. Ramlall and D. Eliezer, "Helix Periodicity, Topology and Dynamics of Membrane-Associated Alpha-Synuclein," Protein Science, Vol. 14, No. 4, 2005, pp. 862-872. doi:10.1110/ps.041255905

[27] W. S. Davidson, A. Jonas, D. F. Clayton and J. M. George, "Stabilization of Alpha-Synuclein Secondary Structure upon Binding to Synthetic Membranes," The Journal of Biological Chemistry, Vol. 273, No. 16, 1998, pp. 9443-9449. doi:10.1074/jbc.273.16.9443

[28] K. A. Conway, J. D. Harper and P. T. Lansbury Jr., "Fibrils Formed in Vitro from Alpha-Synuclein and Two Mutants Forms Linked to Parkinsons's Disease Are Typical Amyloid," Biochemistry, Vol. 39, No. 10, 2000, pp. 2552-2563.

[29] M. Zhu, J. Li and A. L. Fink, "The Association of $\alpha$-Synuclein with Membranes Affects Bilayer Structure, Stability and Fibril Formation," The Journal of Biological Chemistry, Vol. 278, No. 41, 2003, pp. 40186-40197. doi:10.1074/jbc.M305326200

[30] S. Chandra, X. Chen, J. Rizo, R. Jahn and T. C. Sudhof, "A Broken Alpha A Broken Alpha-Helix in Folded AlphaSynuclein," The Journal of Biological Chemistry, Vol. 278, No. 17, 2003, pp. 15313-15318. doi:10.1074/jbc.M213128200

[31] C. M. Pfefferkorn and J. C. Lee, "Tryptophan Probes at the $\alpha$-Synuclein and Membrane Interface," The Journal of Physical Chemistry B, Vol. 114, No. 13, 2010, pp. 46154622. doi:10.1021/jp908092e

[32] V. N. Uversky, J. Li and A. L. Fink, "Evidence for a Partially Folded Intermediate in Alpha-Synuclein Fibril Formation," The Journal of Biological Chemistry, Vol. 276, No. 14, 2001, pp. 10737-10744.
[33] J. C. Lee, R. Langen, P. A. Hummel, H. B. Gray and J. R. Winkler, "Alpha-Synuclein Structures from Fluorescence Energy-Transfer Kinetics: Implications for the Role of the Protein in Parkinson's Disease," Proceedings of the National Academy of Sciences of the United States of America, Vol. 101, No. 47, 2004, pp. 16466-16471. doi:10.1073/pnas.0407307101

[34] D. Sulzer, "Clues to How $\alpha$-Synuclein Damages Neurons in Parkinson's Disease," Movement Disorders, Vol. 25, Suppl. 1, 2010, pp. S27-S31. doi:10.1002/mds.22639

[35] K. Furukawa, et al., "Plasma Membrane Ion Permeability Induced by Mutant $\alpha$-Synuclein Contributes to the Degeneration of Neural Cells," Journal of Neurochemistry, Vol. 97, No. 4, 2006, pp. 1071-1077. doi:10.1111/j.1471-4159.2006.03803.x

[36] B. D. van Rooijen, M. M. A. E. Claessens and V. Subramaniam, "Membrane Binding of Oligomeric Alpha-Synuclein Depends on Bilayer Charge and Packing," FEBS Letters, Vol. 582, No. 27, 2008, pp. 3788-3792. doi:10.1016/j.febslet.2008.10.009

[37] M. T. Stöckl, N. Zijlstra and V. Subramaniam, " $\alpha$-SynuClein Oligomers: An Amyloid Pore?" Molecular Neurobiology, 2012. doi:10.1007/s12035-012-8331-4

[38] M. Stöckl, P. Fischer, E. Wanker and A. Hermann, “Alpha Synuclein Selectively Binds to Anionic Phospholipids Embedded in Liquid-Disordered Domains," Journal of Molecular Biology, Vol. 375, No. 5, 2008, pp. 1394 1404. doi:10.1016/i.jmb.2007.11.051

[39] B. D. van Rooijen, M. M. A. E. Claessens and V. Subramaniam, "Lipid Bilayer Disruption by Oligomeric Alpha-Synuclein Depends on Bilayer Charge and Accessibility of the Hydrophobic Core," Biochim Biophys Acta Biomembr, Vol. 1788, No. 6, 2009, pp. 1271-1278. doi:10.1016/j.bbamem.2009.03.010

[40] M. Stöckl, M. M. Claessens and V. Subramaniam, "Kinetic Measurements Give New Inshights into Lipid Membrane Permeabilization by Alpha-Synuclein Oligomers," Molecular BioSystems, Vol. 8, No. 1, 2012, pp. 338-345. doi: $10.1039 / \mathrm{c} 1 \mathrm{mb} 05293 \mathrm{~d}$

[41] J. E. Shaw, R. F. Epand, R. M. Epand, Z. Li, R. Bittman and C. M. Yip, "Correlated Fluorescence-Atomic Force Microscopy of Membrane Domains: Structure of Fluorescence Probes Determines Lipid Localization," Biophysical Journal, Vol. 90, No. 6, 2006, pp. 2170-2178. doi:10.1529/biophysj.105.073510

[42] M. Stöckl, P. Fischer, E. Wanker and A. Herrmann, " $\alpha$-Synuclein Selectively Binds to Anionic Phospholipids Embedded in Liquid-Disordered Domains," Journal of Molecular Biology, Vol. 375, No. 5, 2008, pp. 1394-1404. doi:10.1016/j.jmb.2007.11.051

[43] B. D. van Rooijen, M. M. A. E. Claessens and V. Subramaniam, "Membrane Permeabilization by Oligomeric $\alpha$-Synuclein: In Search of the Mechanism," Plos One, Vol. 5, No. 12, 2010, Article ID: e14292. doi:10.1371/journal.pone.0014292 